\title{
Report
}

\section{The Epidemiological Survey and Analysis of Canine Babesiosis in Xi'an City, China}

\author{
Wuren $\mathrm{Ma}^{\dagger}$, Yunpeng $\mathrm{Fan}^{\dagger}$, Feng Jie, Lingyu Zhang, Yuan Zhong, Yingqiu Liu, Weimin Zhang, \\ Xiaoping Song*
}

College of Veterinary Medicine, Northwest A\&F University, Yangling, P. R. China

\author{
Email address: \\ sxpxbnl@163.com (Xiaoping Song) \\ ${ }^{*}$ Corresponding author \\ $\dagger$ Wuren Ma and Yunpeng Fan are co-first authors.
}

\section{To cite this article:}

Wuren Ma, Yunpeng Fan, Feng Jie, Lingyu Zhang, Yuan Zhong, Yingqiu Liu, Weimin Zhang, Xiaoping Song. The Epidemiological Survey and Analysis of Canine Babesiosis in Xi' an City, China. Animal and Veterinary Sciences. Vol. 6, No. 3, 2018, pp. 43-50.

doi: 10.11648/j.avs.20180603.12

Received: July 8, 2018; Accepted: July 23, 2018; Published: August 21, 2018

\begin{abstract}
The canine babesiosis is one of the major diseases that significantly harm to the dogs. In order to analyze the characteristics of babesiosis in dogs, and to explore the characteristics of blood changes in canine babesiosis in Xi'an city, 194 cases of canine babesiosis were diagnosed in the animal teaching hospital of the Northwest A\&F University during January 2013 to December 2015. The results showed that autumn was the main season for canine babesiosis in Xi'an city, male dogs under 3 years old were the main pathogenic group, and Teddy dog is the most susceptible breed. In addition, the number of red blood cell, platelet, hematokrit, hemoglobin, eosinophil granulocyte and the concentration of calcium were decreased; the mean corpuscular volume, the number of white blood cell, the concentration of alkaline phosphatase, total bilirubin, urea nitrogen, phosphorus, alanine aminotransferase and aspartate aminotransferase was elevated. Moreover, the liver and kidney function was also destroyed in a certain degree. It indicated that the dogs suffered from severe anemia (regenerative anemia), accompanied by inflammatory reaction, which can cause the blood coagulation dysfunction, some drugs with protecting liver and kidney should be used in the process of treatment so as to improve the cure rate of this disease. These findings provided important reference information for the prevention and treatment of babesiosis.
\end{abstract}

Keywords: Canine Babesiosis, Blood Routine Test, Biochemical Criterion, Case Analysis

\section{Introduction}

Canine babesiosis is the haemal protozoa disease spread by ticks, which is caused by the parasite of babesia in red blood cells, it is one of the major diseases that significantly harm to the dogs ([1-5]). The clinical manifestations of this disease are fever, severe anemia, jaundice, hemoglobinuria, shorter disease course, and higher mortality. This disease has a high morbidity in tick endemic areas, so it is widely popular in Southeast Asia, North America and South America. In our country, the canine babesiosis that has been reported is mostly infected with Babesia gibsoni, which is endemic in some parts of Jiangsu and Henan province. This disease possesses a serious hazard to pet dogs [6].
In 1896, in Africa, there was an earlist description of the parasites in red blood cell of dog associated with the babesiosis of dog [7]. The babesia parasitize in red blood cells of dogs, which could be infected repeatedly by ticks. More than 100 species of babesia have been recorded at present. According to morphology, babesia can be divided into two categories. Large babesia is $3-7 \mu \mathrm{m}$, and small babesia is $1-3$ $\mu \mathrm{m}([8,9])$. Because of the international transport of dogs, new infectious disease could occur once they arrive in new areas. In addition, infected animals exposed to the ticks in new areas may cause new infections. Humans and animals come to a new environment where ticks and reservoir hosts are 
available, they will be exposed in new pathogens. The growing number of mutated babesia is becoming more and more apparent.

The babesia from dog main is the large babesia. According to the differences of genotype, geographical distribution, pathogenicity, media characteristics features, the babesia is divided into three subspecies: B. canis vogeli, B. canis canis and $B$. canis rossi ([10-12]). There are regional differences in the species of babesia. With the development of diagnostic techniques, the babesia from dog has been reclassified. It is difficult to describe the geographical location of the whole babesia of dog, and it is limited by the lack of reports from the application microscope, serology and molecular techniques. With the identification of new species, it is more and more important for clinicians to understand the infection status and epidemic regularity of the babesiosis of dog, and adopt effective diagnosis, prognosis and treatment measures in time. To understand the impact of the canine babesiosis, summary the pathogenesis regularity of babesiosis, and analyze the change rule of key indicators on blood and the function of liver and kidney, the authors analyzed 194 cases diagnosed with the canine babesiosis from the animal teaching hospital of Northwest A\&F university, the aim was to provide the effective diagnosis and treatment methods for preventing and curing the canine babesiosis.

\section{Materials and Methods}

From January 2013 to December 2015, the authors investigated 194 cases diagnosed with the canine babesiosis from the animal teaching hospital of the Northwest A\&F University. The morbidity season, breed, age, gender, blood routine test, and the important biochemical criterion of liver and kidney function were statistically analyzed, such as urea nitrogen (BUN), creatinine (Crea), calcium $(\mathrm{Ca})$, phosphorus (P), alanine aminotransferase (ALT), aspartate aminotransferase (AST) and alkaline phosphatase (ALP), total bilirubin (TB) and so on.

\section{Results}

\subsection{The Overall Morbidity and Analysis}

\subsubsection{The Morbidity Season}

The finding is illustrated in Figure 1. The result showed that the canine babesiosis could occur throughout the year, but winter was the main season of the canine babesiosis, the total number of cases is 74 , which accounted for $38.1 \%$ of the total cases. The number of cases in autumn was 70 , accounting for $36.1 \%$, followed $48(24.7 \%)$ in winter and $2(1.0 \%)$ in spring respectively.

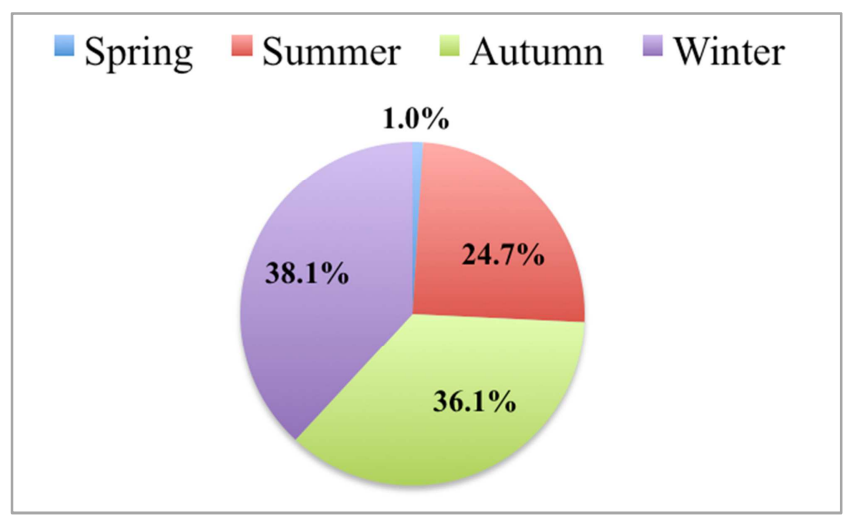

Figure 1. The morbidity season of babesiosis of dog.

\subsubsection{The Breed of Dog}

The result is illustrated in Figure 2. The finding showed that there was a relation between babesiosis and the breed of dog. In all dogs with babesiosis, the number of Teddy was largest, which was 47, the second was Poodle (22). Pomeranian was 15, Mongrel dog was 14, Golden Retriever was 13, Samoyed was 9, Labrador retriever was 8 , German shepherd was 7 , and Bichon Frise was 6. Schnauzer, Belgian Malinois, Border collie and Rough Collie were all 4. Chihuahuas and Husky were all 3. Akitas dog, Alaskan malamute, Pug and Shetland sheepdog were all 2. Other breed was 10 .

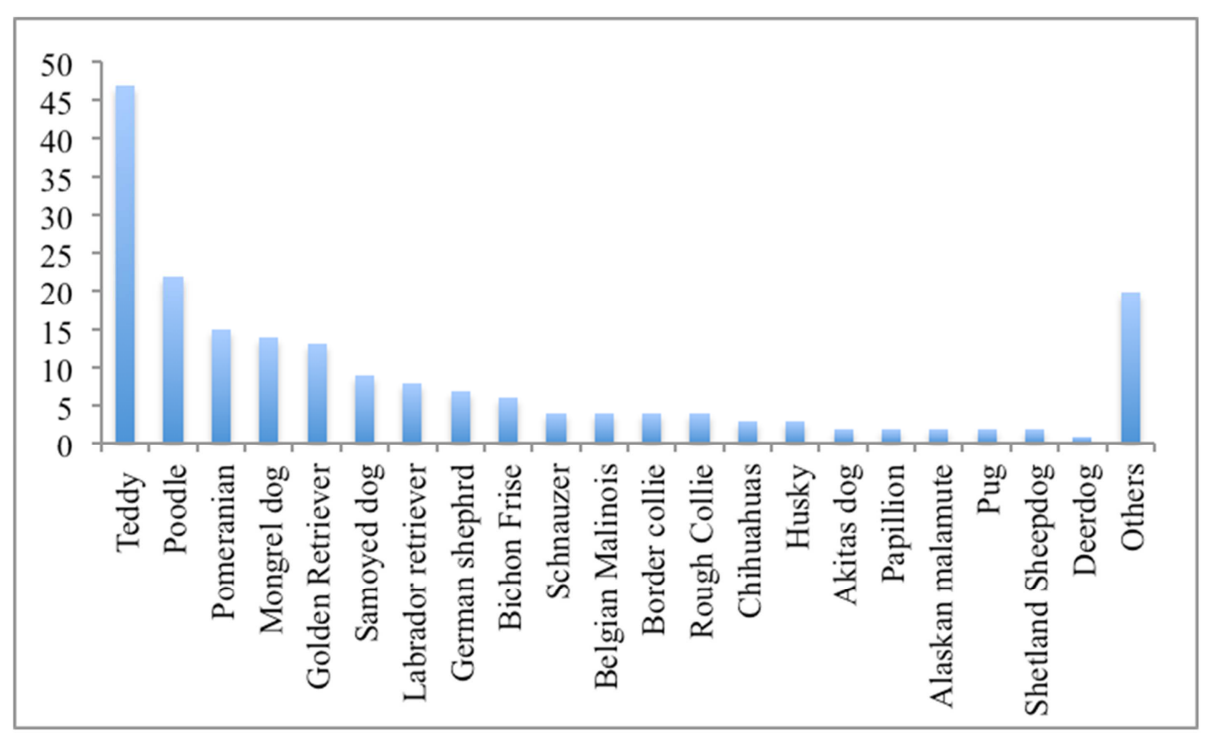

Figure 2. The breed of dogs with babesiosis. 


\subsubsection{The Age of Onset}

The age of onset is shown in Figure 3. The lowest age of the dogs occurred babesiosis was 3 months old, and the highest was 13 years old. There were 129 cases under 4 years old, accounting for $66.4 \%$ of the total number of cases. The number of dogs was 54 from 4 to 13 years old, which accounted for $33.6 \%$.

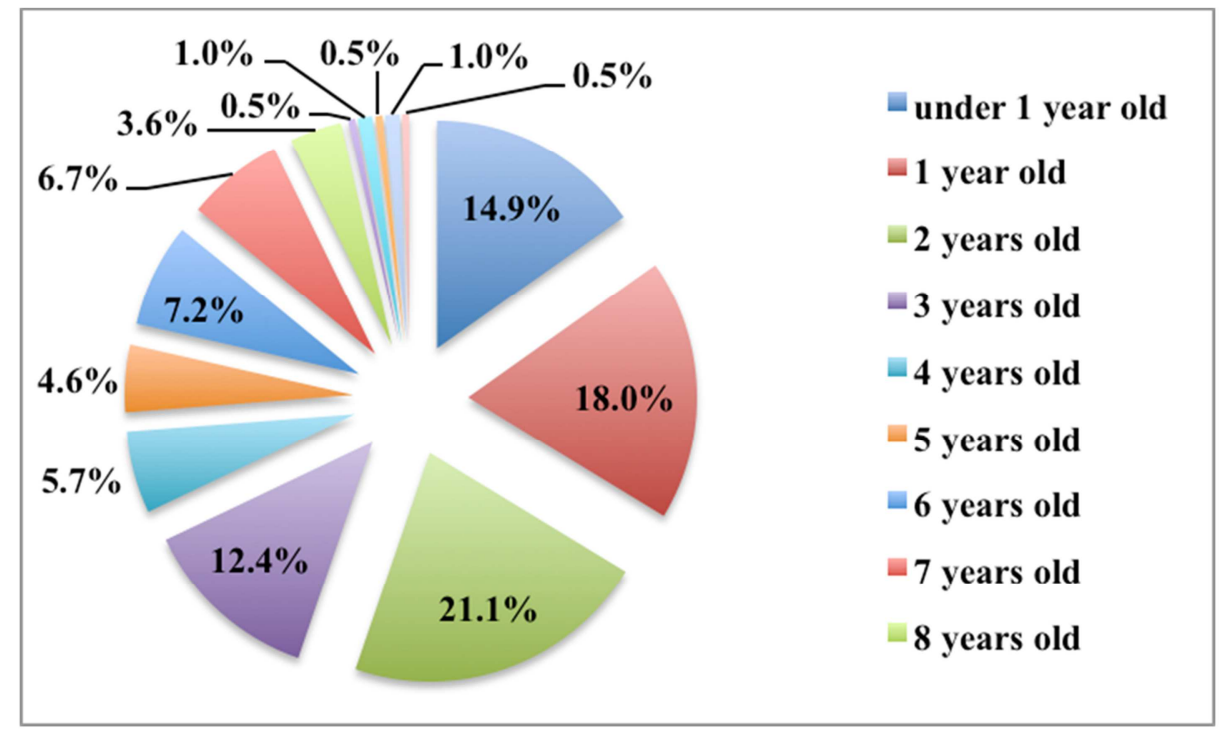

Figure 3. The age of onset with babesiosis.

\subsubsection{The Gender of Dog}

The gender of dogs with babesiosis is shown in Figure 4. Most dogs were male, which accounted for $62.4 \%$ of the total number of cases. The percentage of female dogs was $37.6 \%$.

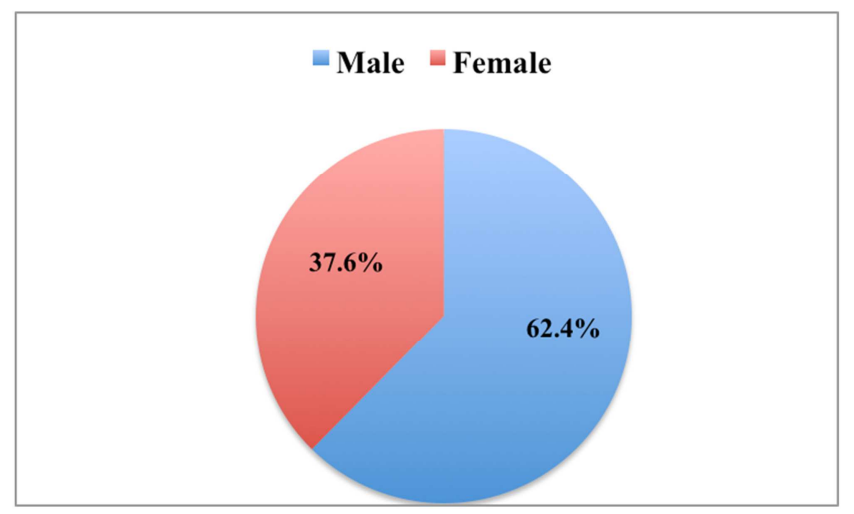

Figure 4. The gender of dogs with babesiosis.

\subsection{The Result of Blood Routine Test}

The result of blood routine test is listed in Table 1. The red blood cells (RBC), hematokrit (HCT), hemoglobin (HGB), eosinophil granulocyte (EO) and platelet (PLT) were decreasing in all cases, and they were lower than reference value (RV), which accounted for $100 \%, 100 \%, 100 \%, 68.47 \%$ and $94.6 \%$ of the total survey cases respectively. The mean corpuscular volume (MCV) was slightly elevatory, and the percentage was $74.77 \%$ in all cases, which was higher than RV.

In addition, the total number of white blood cells (WBC) was slightly elevatory, accounting for $59.46 \%$ of the total cases within RV, $37.84 \%$ was higher than RV, and only $2.70 \%$ was lower than RV. In the classification of WBC, the average of neutrophils (NEU) was higher than RV, which accounted for $39.64 \%$ of the total cases. The percentage was $55.86 \%$ within the scope of RV, and only $4.50 \%$ was lower than RV. The average of Lymphocyte (LYM), mononuclear (MON) and basophils (BASO) was 4.46, 0.98 and 0.03 respectively, which all lied in the scope of the RV, the cases accounted for $68.46,81.98 \%$ and $93.70 \%$ of the total number of investigation cases, the percentage which was higher than RV was $31.53 \%, 18.02 \%$ and $2.70 \%$ respectively, and there was no any cases which was lower than RV.

Table 1. The result of blood test in dogs with babesiosis.

\begin{tabular}{|c|c|c|c|c|c|c|c|}
\hline Item & Reference value & Average value & Maximum value & Minimum value & $<\mathbf{R V}$ & $>\mathbf{R V}$ & Within RV \\
\hline $\operatorname{RBC}\left(10^{12} / \mathrm{L}\right)$ & $5.5 \sim 8.5$ & 2.08 & 4.81 & 0.55 & $100 \%$ & 0 & 0 \\
\hline $\mathrm{HGB}(\mathrm{g} / \mathrm{L})$ & $120 \sim 180$ & 47.89 & 111 & 13.2 & $100 \%$ & 0 & 0 \\
\hline HCT (\%) & $37 \sim 55$ & 17.65 & 33.8 & 6.9 & $100 \%$ & 0 & 0 \\
\hline $\mathrm{WBC}\left(10^{12} / \mathrm{L}\right)$ & $6 \sim 17$ & 18.30 & 71.13 & 3.79 & $2.70 \%$ & $37.84 \%$ & $59.46 \%$ \\
\hline NEU $\left(10^{9} / \mathrm{L}\right)$ & $3 \sim 11.5$ & 12.72 & 61.67 & 1.81 & $4.50 \%$ & $39.64 \%$ & $55.86 \%$ \\
\hline LYM $\left(10^{9} / \mathrm{L}\right)$ & $1 \sim 4.8$ & 4.46 & 10.68 & 1.09 & 0 & $31.53 \%$ & $68.46 \%$ \\
\hline
\end{tabular}




\begin{tabular}{llllllll}
\hline Item & Reference value & Average value & Maximum value & Minimum value & $<$ RV & $>$ RV & Within RV \\
\hline EO $\left(10^{9} / \mathrm{L}\right)$ & $0.1 \sim 1.25$ & 0.09 & 1.09 & 0 & $68.47 \%$ & 0 & $31.53 \%$ \\
BASO $\left(10^{9} / \mathrm{L}\right)$ & $0 \sim 0.1$ & 0.03 & 0.9 & 0 & 0 & $2.70 \%$ & $93.70 \%$ \\
PLT $\left(10^{9} / \mathrm{L}\right)$ & $150 \sim 500$ & 52.19 & 647 & 2 & $94.60 \%$ & $0.90 \%$ & $4.50 \%$ \\
\hline
\end{tabular}

RBC, Red blood cells; HGB, hemoglobin; HCT, hematocrit; MCV, mean corpuscular volume; WBC, white blood cell; NEU, neutrophils; LYM, Lymphocyte; MON, mononuclear; EO, eosinophil; BASO, basophils; PLT, platelet; RV, reference value.

\subsection{The Impact of Canine Babesiosis on the Function of Liver and Kidney}

The changes of main biochemical criterion in liver and kidney are listed in Table 2. The results showed that this disease could affect the main biochemical criterion related to the function of liver and kidney. In these indexes, the concentration of calcium (Ca) in blood was almost decreased in cases compared with RV, accounting for $82.8 \%$ of the total amount of cases. Other indexes were increased, and hardly any case was lower than RV. The concentration of alkaline phosphatase (ALP) was higher than RV, which accounted for $87.9 \%$ of the total amount of cases, the second was total bilirubin (TB), accounting for $56.0 \%$. The percentage of blood urea nitrogen (BUN), phosphorus $(\mathrm{P})$, alanine aminotransferase (ALT) and aspartate aminotransferase (AST) was $25.9 \%, 24.1 \%, 21.6 \%$ and $28.4 \%$ respectively, which were all higher than RV.

Table 2. The changes of main biochemical criterion in liver and kidney.

\begin{tabular}{|c|c|c|c|c|c|c|c|c|}
\hline & BUN (mmol/L) & $\mathrm{Cr}(\mu \mathrm{mol} / \mathrm{L})$ & $\mathrm{Ca}(\mathrm{mmol} / \mathrm{L})$ & $\mathrm{P}(\mathrm{mmol} / \mathrm{L})$ & $\operatorname{ALT}(\mathbf{U} / \mathbf{L})$ & AST (U/L) & $\operatorname{ALP}(\mathbf{U} / \mathbf{L})$ & TB $(\mu \mathrm{mol} / \mathrm{L})$ \\
\hline RV & $2.9-9.6$ & $36-124$ & $2.57-3.03$ & $0.61-1.61$ & $5-80$ & $10-80$ & $10-254$ & $0-15$ \\
\hline Number of dogs above RV & 30 & 11 & 2 & 28 & 25 & 33 & 102 & 65 \\
\hline Percentage $(\%)$ & 25.9 & 9.5 & 1.7 & 24.1 & 21.6 & 28.4 & 87.9 & 56.0 \\
\hline Number of dogs under RV & 1 & 2 & 96 & 1 & 1 & 0 & 0 & 0 \\
\hline Percentage $(\%)$ & 0.9 & 1.7 & 82.8 & 0.9 & 0.9 & 0.0 & 0.0 & 0.0 \\
\hline
\end{tabular}

BUN, urea nitrogen; Crea, creatinine; Ca, calcium; P, phosphorus; ALT, alanine aminotransferase; AST, aspartate aminotransferase; ALP, alkaline phosphatase; $\mathrm{TB}$, total bilirubin.

\subsection{The Characteristic of Cases with Elevated ALP}

The concentration of alkaline phosphatase (ALP) was higher than RV, which accounted for $87.9 \%$ of the total amount of cases. The result is showed in Table 3. In breed aspect of sick dogs, Teddy was the highest, accounting for $35.3 \%$. The second was Mongrel dog, which accounted for $12.7 \%$ of the total cases. The followed was Pomeranian and
Golden Retriever, which accounted for $7.8 \%$ and $5.9 \%$ respectively. In age aspect of cases, the percentage was the highest when the age of dogs was 1 to 3 years old, which was $39.2 \%$. The percentage was $23.5 \%$ when the age of dogs was no more than 1year old. In gender aspect of cases, the total number of male dog was 63 , which accounted for $61.8 \%$, the percentage of female dog was $38.2 \%$.

Table 3. The characteristic of cases with elevated ALP.

\begin{tabular}{lllll}
\hline Breed & Teddy & Mongrel dog & Pomeranian & Golden Retriever \\
\hline Number & 36 & 13 & 8 & 6 \\
Percentage (\%) & 35.3 & 12.7 & 7.8 & 5.9 \\
Age & $\leq 1$ year old & $1-3$ years old & $3-6$ years old & $>6$ years old \\
Number & 24 & 40 & 17 & 21 \\
Percentage (\%) & 23.5 & 39.2 & 16.7 & \\
Sex & Male & Female & & \\
Number & 63 & 39 & & \\
Percentage (\%) & 61.8 & 38.2 & & \\
\hline
\end{tabular}

\subsection{The Characteristic of Cases with Elevated TB}

The result is showed in Table 4. The number of dogs with elevated TB was 65 , accounting for $56 \%$ of the total number of cases. In breed aspect of sick dogs, Teddy was the highest, accounting for $29.2 \%$. The second was Mongrel dog, which accounted for $10.8 \%$ of the total cases. The followed was
Golden Retriever and Pomeranian, which accounted for 9.2\% and $7.7 \%$ respectively. In age aspect of cases, the percentage was the highest when the age of dogs was 1 to 3 years old, which was $35.4 \%$. The percentage was $23.1 \%$ when the age of dogs was larger than 6 years old. In gender aspect of cases, the total number of male dog was 43 , which accounted for $66.2 \%$, the percentage of female dog was $33.8 \%$.

Table 4. The characteristic of cases with elevated TB.

\begin{tabular}{lllll}
\hline Breed & Teddy & Mongrel dog & Golden Retriever \\
\hline Number & 19 & 7 & 6 & Pomeranian \\
Percentage (\%) & 29.2 & 10.8 & 9.2 & 5 \\
Age & $\leq 1$ year old & $1-3$ years old & $3-6$ years old & 7.7 \\
Number & 13 & 23 & 14 & $>6$ years old \\
Percentage (\%) & 20 & 35.4 & 21.5 & 23.1 \\
\hline
\end{tabular}




\begin{tabular}{lllll}
\hline Breed & Teddy & Mongrel dog & Golden Retriever & Pomeranian \\
\hline Sex & Male & Female & & \\
Number & 43 & 22 & \\
Percentage (\%) & 66.2 & 33.8 & \\
\hline
\end{tabular}

\subsection{The Characteristic of Cases with Elevated AST}

The result is showed in Table 5. The number of dogs with elevated AST was 33, accounting for $28.4 \%$ of the total number of cases. In breed aspect of sick dogs, Mongrel dog was the highest, accounting for $27.3 \%$. The second was Teddy, which accounted for $21.2 \%$ of the total cases. The followed was German shepherd, which accounted for $12.1 \%$. In age aspect of cases, the percentage was the highest when the age of dogs was 1 to 3 years old, which was $36.4 \%$. The percentage was $30.3 \%$ when the age of dogs was larger than 6 years old. In gender aspect of cases, the total number of male dog was 19, which accounted for $57.6 \%$, the percentage of female dog was $42.4 \%$.

Table 5. The characteristic of cases with elevated AST.

\begin{tabular}{|c|c|c|c|c|}
\hline Breed & Mongrel dog & Teddy & German shepherd & Pomeranian \\
\hline Number & 9 & 7 & 4 & 2 \\
\hline Percentage (\%) & 27.3 & 21.2 & 12.1 & 6.1 \\
\hline Age & $\leq 1$ year old & 1-3 years old & 3-6 years old & $>6$ years old \\
\hline Number & 5 & 12 & 6 & 10 \\
\hline Percentage (\%) & 15.2 & 36.4 & 18.2 & 30.3 \\
\hline Sex & Male & Female & & \\
\hline Number & 19 & 14 & & \\
\hline
\end{tabular}

\subsection{The Characteristic of Cases with Hypocalcemia}

The result is showed in Table 6 . The number of dogs with hypocalcemia was 96 , accounting for $82.8 \%$ of the total number of cases. In breed aspect of sick dogs, Teddy was the highest, accounting for $35.4 \%$. The second was Mongrel dog, which accounted for $12.5 \%$ of the total cases. The followed was Pomeranian and Golden Retriever, which all accounted for $6.3 \%$. In age aspect of cases, the percentage was the highest when the age of dogs was 1 to 3 years old, which was $38.5 \%$. The percentage was $20.8 \%$ when the age of dogs was 3 to 6 years old. In gender aspect of cases, the total number of male dog was 63 , which accounted for $65.6 \%$, the percentage of female dog was $34.4 \%$.

Table 6. The characteristic of cases with hypocalcemia.

\begin{tabular}{|c|c|c|c|c|}
\hline Breed & Teddy & Mongrel dog & Pomeranian & Golden Retriever \\
\hline Number & 34 & 12 & 6 & 6 \\
\hline Percentage (\%) & 35.4 & 12.5 & 6.3 & 6.3 \\
\hline Age & $\leq 1$ year old & 1-3 years old & 3-6 years old & $>6$ years old \\
\hline Number & 18 & 37 & 20 & 21 \\
\hline Percentage (\%) & 18.8 & 38.5 & 20.8 & 21.9 \\
\hline Sex & Male & Female & & \\
\hline Number & 63 & 33 & & \\
\hline
\end{tabular}

\subsection{The Characteristic of Cases with Elevated BUN}

The result is showed in Table 7. The number of dogs with elevated BUN was 30, accounting for $25.9 \%$ of the total number of cases. In breed aspect of sick dogs, Teddy was the highest, accounting for $43.3 \%$. The second was Mongrel dog, which accounted for $16.7 \%$ of the total cases. The third was
Pomeranian, which accounted for $10.0 \%$. In age aspect of cases, the percentage was the highest when the age of dogs was 1 to 3 years old, which was $33.3 \%$. The percentage was $30.0 \%$ when the age of dogs was larger than 6 years old. In gender aspect of cases, the total number of male dog was 19 , which accounted for $63.3 \%$, the percentage of female dog was $36.7 \%$.

Table 7. The characteristic of cases with elevated BUN.

\begin{tabular}{|c|c|c|c|c|}
\hline Breed & Teddy & Mongrel dog & Pomeranian & German shepherd \\
\hline Number & 13 & 5 & 3 & 1 \\
\hline Percentage (\%) & 43.3 & 16.7 & 10 & 3.3 \\
\hline Age & $\leq 1$ year old & $1-3$ years old & 3-6 years old & $>6$ years old \\
\hline Number & 6 & 10 & 5 & 9 \\
\hline Percentage (\%) & 20 & 33.3 & 16.7 & 30 \\
\hline Sex & Male & Female & & \\
\hline Number & 19 & 11 & & \\
\hline
\end{tabular}




\subsection{The Characteristic of Cases with Hyperphosphatemia}

The result is showed in Table 8. The number of dogs with hyperphosphatemia was 28 , accounting for $24.1 \%$ of the total number of cases. In breed aspect of sick dogs, Teddy was the highest, accounting for $17.9 \%$. The second was Mongrel dog, which accounted for $14.3 \%$ of the total cases. The third was German shepherd, which accounted for $10.7 \%$. In age aspect of cases, the percentage was the highest when the age of dogs was no more than 1year old, which was $35.7 \%$. The percentage was $32.1 \%$ when the age of dogs was 1 to 3 years old. The percentage was $21.4 \%$ when the age of dogs was larger than 6 years old. In gender aspect of cases, the total number of male dog was 17 , which accounted for $60.7 \%$, the percentage of female dog was $39.3 \%$.

Table 8. The characteristic of cases with hyperphosphatemia.

\begin{tabular}{lllll}
\hline Breed & Teddy & Mongrel dog & German shepherd & Pomeranian \\
\hline Number & 5 & 4 & 3 & 2 \\
Percentage (\%) & 17.9 & 14.3 & 10.7 & 7.1 \\
Age & $\leq 1$ year old & $1-3$ years old & 3 -6 years old & $>6$ years old \\
Number & 10 & 9 & 3 & 6 \\
Percentage (\%) & 35.7 & 32.1 & 10.7 & 21.4 \\
Sex & Male & Female & & \\
Number & 17 & 11 & & \\
Percentage (\%) & 60.7 & 39.3 & & \\
\hline
\end{tabular}

\section{Discussion}

This survey was completed at the animal teaching hospital of Northwest A\&F University for three years with a certain representative. The survey results showed that the morbidity of babesiosis in autumn and winter is the highest in Xi'an city Shaanxi province, which accounted for $74.2 \%$ of the total number of cases. Babesiosis is spread through ticks, which could occur by the bite of tick infected with babesia. In Xi'an city, and the activity of dogs in outdoor is frequent in autumn, at the same time, ticks also frequently haunt in the lawn. Therefore, the dogs usually infect babesiosis when they hang out in the lawn, and the parasitic tick could often be detected on the skin of sick dogs [13]. This result indicates that the dog needs to be dewormed in vitro before getting outside, especially in the autumn with the frequent activity of ticks.

The survey results showed that the dogs with babesiosis was young, the percentage was $66.4 \%$ of the total cases under 4 years old. The male dogs were much easier to infect babesiosis, accounting for $66.4 \%$ of the total number of cases. In addition, in all dogs with babesiosis, the number of Teddy was largest, which accounted for $24.2 \%$. There is no research report about the etiology of babesiosis on the age, gender and breed at home and abroad, and it needs to be further studied. It is reported that the bite may also cause babesiosis through ticks contact with the blood directly to be transmitted, which does not need media to be transmitted $([14,15])$. Just for reason as above, the monitoring of the epidemiology of babesia should be strengthened.

The main function of red blood cells is to transport oxygen and carbon dioxide for metabolism of body, and play an important role in maintaining homeostasis. In the blood, 98.5\% of oxygen is transported by binding to the hemoglobin [6]. Therefore, the concentration of hemoglobin is the most direct indicator for measuring the oxygen carrying capacity of blood. All cases of mucosa pale appeared severe anemia, and the degree of anemia is related with the length of the disease. The anemia of sick dogs is extremely serious with acting depressed for more than a week. The reason may be that pathogens replicate in the red blood cells, and then cause intravascular and extravascular hemolytic anemia $([14,16])$. The survey results showed that the number of red blood cells of all dogs with babesiosis decreased at different levels, and it went down to $0.55 \times 10^{12} / \mathrm{L}$ in sickest dogs. There were 63 cases, the red blood cells of whom were less than $2 \times 10^{12} / \mathrm{L}$, accounting for $56.76 \%$, which is characterized with severe anemia. At the same time, the concentration of hemoglobin and hematokrit were decreased at different degrees. It indicated that the red blood cells were destroyed when the dogs infected with babesia. There were 71 cases that the hematocrit was less than $20 \%$, accounting for $63.96 \%$ of the total cases, it suggested that the sick dog should be treated by blood transfusion in time. In addition, the percentage was $74.77 \%$ in all cases that the mean corpuscular volume was beyond the reference value. It indicated that the immature red blood cells (namely reticulocyte) increased in the blood circulation, it means that the dogs with babesiosis all had reproducible anemia, and it don't need to consider the treatment of aplastic anemia.

The parasite possesses the complex life history and varied form, which can cause complicated immune response. After infected by babesia, host can generate the anti-red cell membrane antibody combined with autoantigen, which enhances the phagocytosis of macrophage on red blood cells and causes immune-mediated hemolytic anemia, and further aggravate the anaemic symptom of dogs with babesiosis [17]. In this survey, a large number of cases possessed the immune-mediated hemolytic anemia, which not only induced severe anemia, but also caused the compensatory action of bone marrow, and increased the number of peripheral blood leukocyte (with nuclear left shift). The result of blood test showed that the percentage of cases with leukocytosis, neutrophilic leukocytosis, lymphocytosis and mononucleosis was $37.84 \%, 19.02 \%, 31.53 \%$ and $39.64 \%$ respectively. It indicated that babesiosis could induce the change of blood and cause anemia in dogs. 
Babesiosis can cause severe tissue hypoxia, and then induce the widespread tissue destruction and the release of inflammatory mediators, which leads to the happening of inflammation [18]. Excessive release of inflammatory media could cause the systemic inflammatory response syndrome. Blood routine examination showed leukocytosis or leukopenia with nuclear left metastasis. Many literatures proved that the number of eosinophils would be increased after infected by babesia ([17, 19]). However, this survey found that the eosinophils is decreased in $68.47 \%$ of all cases, others was within in the normal range, there was no any case with eosinophilia. It needs to further investigate whether this result is related to the severe anemia of dogs, the long infection time, and other reasons. Blood coagulation function is related to the activity of platelet. When the dog is infected by babesia, the platelets decrease dramatically. It is reported that the dogs infected with babesia only had symptom of disseminated intravascular coagulation, but the obvious hemorrhagic symptoms is less prevalent in clinic [20]. In this survey, the platelets of the dogs significantly reduced, the number of platelets in 105 dogs was decreased at different levels, accounting for $94.59 \%$. In addition, the number of male dogs was 63 , female was 42 . Thrombocytopenia could occur alone or simultaneous with hematological abnormalities, which may be transient or persistent, and is usually associated with abnormal blood coagulation [21]. Thrombocytopenia can be used as a clinical feature for diagnosing the babesiosis of dog. The reason of thrombocytopenia may be related to the immune or coagulation loss of platelets induced by hemolysis or vascular injury. Because canine babesiosis could cause the hemolytic anemia, then activate the original thrombin system, lead to excessive consumption of platelet, so the number of platelets severely decreases [22]. Since the sick dogs had no obvious bleeding, the most likely reason is the immune-mediated anemia.

The survey found that canine babesiosis could affect the main indicators of liver and kidney at different degrees, suggesting that babesiosis could cause the damage of liver and kidney function. It implied that some drugs for protecting liver and kidney function should be used when curing this disease, in order to prevent the further injury of the liver and kidney. The result showed that the most affected is alkaline phosphatase among these indicators. Babesiosis could increase the content of alkaline phosphatase in the blood, there were 102 cases, accounting for $87.9 \%$ of the total cases. The second is calcium, babesiosis could cause hypocalcemia, and the percentage was $82.8 \%$. It indicated that calcium needed to be supplied in the treatment of babesiosis. After crosswise compared the main indicators of liver and kidney function, the result showed that the number of Teddy dog with abnormal indicators was the largest. It indicated that Teddy was not only susceptible breed, but also its liver and kidney function affected by babesiosis is the biggest. Among all these indicators, the number of dogs with babesiosis was the highest from 1 to 3 years old, while the other ages were relatively average. In addition, the number of the male dogs with babesiosis was the largest, indicating that male dogs possessed the less tolerant for this disease. Therefore, special attention should be paid to male dogs in clinical treatment.

\section{Conclusion}

The survey result showed that autumn was the main season for canine babesiosis in Xi'an city, and the young males dogs under 3 years old were the main pathogenic group, and Teddy dog is the most susceptible breed, and the damage of liver and kidney function was also the most serious. In addition, babesiosis could cause renewable anemia of dogs, and had a certain degree of inflammation. These findings provided important reference information for the prevention and treatment of babesiosis. It is suggested that some drugs should be used in the process of treatment so as to improve the cure rate of this disease.

\section{Acknowledgements}

The project was supported by a grant from the Fundamental Research Funds for the Central Universities (Z109021606)

\section{Abbreviations}

BUN, urea nitrogen; Crea, creatinine; $\mathrm{Ca}$, calcium; $\mathrm{P}$, phosphorus; ALT, alanine aminotransferase; AST, aspartate aminotransferase; ALP, alkaline phosphatase; TB, total bilirubin; RBC, red blood cells; HCT, hematocrit; HGB, hemoglobin; EO, eosinophil granulocyte; PLT, platelet; RV, reference value; $\mathrm{MCV}$, mean corpuscular volume; WBC, white blood cells; NEU, neutrophils; LYM, Lymphocyte; MON, mononuclear; BASO, basophils.

\section{References}

[1] Abdullahi S U, Mohammed A A, Trimnell A R, et al., 1990. Clinical and haematological findings in 70 naturally occurring cases of canine babesiosis. Journal of Small Animal Practice. $31,145-147$.

[2] Kuleš J, Torre-Minguela C, Barić R R, et al., 2016. Plasma biomarkers of SIRS and MODS associated with canine babesiosis. Research in Veterinary Science. 105, 222-228.

[3] Josipa K, Jelena G, Vladimir M, et al., 2017. Alteration of haemostatic parameters in uncomplicated canine babesiosis. Comparative Immunology Microbiology and Infectious Disease. 53, 1-6.

[4] Gad Baneth, 2018. Antiprotozoal treatment of canine babesiosis. Veterinary Parasitology. 254, 58-63.

[5] Shekerah P, Lavoisier A, Samantha S, et al., 2018. Screening of patient blood samples for babesiosis using enzymatic assays. Ticks and Tick-borne Disease. 9, 302-306.

[6] Birkenheuer A J, Levy M G, Stebbins M, 2003. Atovaquone and azithromycin combination therapy for the treatment of Babesia gibsoni infections in dogs. Journal Veterinary Internal Medicine. 17, 381-382.

[7] Kong F Y, 1997. Animal parasitology [M]. Beijing: China agricultural university press. $315-316$. 
[8] Hou J F, 2002. Small animal diseases. [M]. Beijing: China agricultural university press. 182-184.

[9] Greene C E, 2012. Infectious diseases of the dog and cat. Saunders; Elsevier Inc. 773-775.

[10] Welzl C, Leisewitz A L, Jacobson L S, et al., 2001. Systemic inflammatory response syndrome and multiple-organ damage/dysfunction in complicated canine babesiosis. Journal of the South African Veterinary Association-tydsrif Vandie Suid-a 72, 158-162.

[11] Matijatko V, Torti M, Schetters T P, 2012. Canine babesiosis in Europe: how many diseases? Trends in Parasitology. 28, 99105.

[12] Wojciech Z, Olga G Z, Piotr B, et al., 2015. Low T3 syndrome in canine babesiosis associated with increased serum IL-6 concentration and azotaemia. Veterinary Parasitology. 211, 2327.

[13] Michael L, Georges K, Alexander T, et al., 2008. Seasonal occurrence of canine babesiosis is influenced by local climate conditions. International Journal of Medicine and Microbiology. 298, 243-248.

[14] Birkenheuer A J, Levy M G, Stebbins M, et al., 2003. Serosurvey of anti Babesia antibodies in stray dogs and American pit bull terriers and American Staffordshire terriers from North Carolina. Journal of the American Animal Hospital Association. 39, 551-557.

[15] Vesna M, Ivana K, Marin T, et al., 2009. Septic shock in canine babesiosis. Veterinary Parasitology. 162, 263-270.
[16] Defauw P, Schoeman J P, Smets P, et al., 2012. Assessment of renal dysfunction using urinary markers in canine babesiosis caused by Babesia rossi. Veterinary Parasitology. 190, 326332 .

[17] Birkenheuer A J, Neel J, Ruslander D, et al., 2004. Detection and molecular characterization of a novel large Babesia species in a dog. Veterinary Parasitology. 124, 151-160.

[18] Amelia G, Bo W, Johan P, 2013. Schoeman, Annemarie T. Kristensen, Mads Kjelgaard-Hansen. Mortality in virulent canine babesiosis is associated with a consumptive coagulopathy. Veterinary Journal. 196, 213-217.

[19] Bohm M, 2006. The comparative assessment of Babesia rossi capillary and venous blood parasitaemias on thin blood smears and their association with disease manifestations. Thesis, University of Pretoria, South Africa.

[20] Boozer A L and Macintire D K, 2003. Canine babesiosis. Veterinary Clinics of North American Small Animal Practice. 33, 885-904.

[21] Bosman A M, Venter E H, Penzhorn B L, 2006. Occurrence of Babesia felis and Babesia leo in various wild felid species and domestic cats in Southern Africa, based on reverse line blot analsis. Veterinary Parasitology. 144, 33-38.

[22] Bourdoiseau G, 2006. Canine babesiosis in France. Veterinary Parasitology, 138, 118-125. 